\title{
XLVII. Supplementary table for computing the precession and nutation of the fixed stars
}

\section{F. Baily Esq. F.R.S. L.S.}

To cite this article: F. Baily Esq. F.R.S. L.S. (1823) XLVII. Supplementary table for computing the precession and nutation of the fixed stars, Philosophical Magazine Series 1, 61:299, 217-218, DOI: $10.1080 / 14786442308644304$

To link to this article: http://dx.doi.org/10.1080/14786442308644304

曲 Published online: 29 Jul 2009.

Submit your article to this journal $\sqsubset \pi$

Џ Article views: 2

Q View related articles $\sqsubset$ 
$142^{\prime \prime}$, the correct difference of altitude resulting from observations at five different stations, $64 \frac{1}{9}$ feet. The true angular difference is consequently $2^{\prime} 36^{\prime \prime}$; the refraction at Ingleborough $\frac{1}{16}$; and at Great Whernside $\frac{1}{15}$.

X. X.

[To be continued.]

XLVII. Supplementary Table for computing the Precession and Nutation of the Fixed Stars. By F. BAILY, Esq. F.R.S. and L.S.

\section{To the Editors of the Philosophical Magazine and Journal.}

IN your Magazine for October last, I alluded to some new 1 tables of Aberration and Nutation which had been computed under the direction of $\mathrm{M}$. Schumacher, and inserted by him in his Astronomische Hiilfstafeln for 1822. Tables for the computation of those quantities are of constant use to the practical astronomer: but, it must be evident, to those who are acquainted with the principles on which M. Schumacher's tables are constructed, that the values denominated $\mathbf{A}$ and $\mathbf{B}$ (which are confined to the Precession and Nutation) require to be calculated every year. M. Schumachex, however, has given them only for the years 1819-1822; which renders the general tables, to which they refer, of little or no use at the present time.

In order to remedy this temporary defect, I have computed (for my own use) the logarithms of $A$ and $B$. for the years 1823 and 1824: and as they may probably be of service to some of your readers, and perhaps render the adoption of M. Schumacher's tables more general, I have sent them for insertion in your Magazine. I should remark that, in the value of $B$, I have included the quantity denoted by $+\cdot 08768$ $\cos 28$, in order that the total result may accord with those deduced from the rigorous formula given by M.'Bessel.

I am, gentlemen,

Your obedient servant,

March 19, 1823.

Francis Baily.

Vol. 61. No. 299. March 1823.

$\mathrm{Ee}$

Day. 
218 Table for the Precession and Nutation of the Fixed Stars.

\begin{tabular}{|c|c|c|c|c|c|}
\hline \multirow{2}{*}{\multicolumn{2}{|c|}{ Day. }} & \multicolumn{2}{|c|}{1823.} & \multicolumn{2}{|c|}{$18 \% 4}$. \\
\hline & & Log. A. & Log. B. & Log. A. & Log. B. \\
\hline \multirow[t]{4}{*}{ January } & 0 & $9 \cdot 4314$ & $0 \cdot 7042^{n}$ & $9 \cdot 5104$ & $0.3924 n$ \\
\hline & 10 & $9 \cdot 4876$ & $0 \cdot 7075^{n}$ & $9 \cdot 5567$ & $0.3969 n$ \\
\hline & 20 & $9 \cdot 5331$ & $0 \cdot 7152 n$ & $9 \cdot 5958$ & $0.4103 n$ \\
\hline & 30 & 9.5735 & $0 \cdot 7257 n$ & $9 \cdot 6290$ & $0.4292 n$ \\
\hline \multicolumn{2}{|c|}{ February 9} & $9 \cdot 6057$ & $0.7368 n$ & $9 \cdot 6568$ & $0.4489 n$ \\
\hline & 19 & $9 \cdot 6326$ & $0.7465 n$ & $9 \cdot 6797$ & $0 \cdot 4657 n$ \\
\hline \multirow[t]{4}{*}{ March } & 1 & $9 \cdot 6553$ & $0.7532 n$ & $9 \cdot 6994$ & $0.4765 n$ \\
\hline & 11 & $9 \cdot 6750$ & $0.7555 n$ & $9 \cdot 7165$ & $0.4791 t$ \\
\hline & 21 & $9 \cdot 6931$ & $0.7529 n$ & $9 \cdot 7322$ & $0 \cdot 4722 n$ \\
\hline & 31 & $9 \cdot 7105$ & $0.7449 n$ & $9 \cdot 7474$ & $0.4549 n$ \\
\hline \multirow[t]{3}{*}{ April } & 10 & $9 \cdot 7278$ & $0.7318_{11}$ & 630 & $267 n$ \\
\hline & 20 & $9 \cdot 7466$ & $0 \cdot 7136_{n}$ & $9 \cdot 7793$ & $75 n$ \\
\hline & 30 & $9 \cdot 7670$ & $0.6919 n$ & $9 \cdot 7969$ & $0.3378 n$ \\
\hline \multirow[t]{3}{*}{ May } & 10 & $9 \cdot 7878$ & $95 n$ & $9 \cdot 8156$ & $0.2832_{n}$ \\
\hline & 20 & $9^{\circ}$ & $0 \cdot 6436_{n}$ & 356 & $0 \cdot 2142_{n}$ \\
\hline & 30 & $9 \cdot 8322$ & $0 \cdot 6209_{n}$ & $9 \cdot 8561$ & $0 \cdot 1472 n$ \\
\hline \multirow[t]{3}{*}{ June } & 9 & $9 \cdot 8.547$ & $0.6022_{n}$ & $9 \cdot 8770$ & $0.0852_{n}$ \\
\hline & 19 & $9 \cdot 8768$ & $0 \cdot 5893_{n}$ & $9 \cdot 8975$ & $0.0367_{n}$ \\
\hline & 29 & $9 \cdot 8980$ & 0.5 & $9 \cdot 9171$ & $0.0101_{n}$ \\
\hline \multirow[t]{3}{*}{ July } & 9 & $9 \cdot 9178$ & $0.5839 n$ & 9.9356 & $0.0081_{n}$ \\
\hline & 19 & 5.3 & 0.5 & 526 & $86 u$ \\
\hline & 29 & $9 \cdot 9515$ & $0.6011_{n}$ & $9 \cdot 9677$ & $626 n$ \\
\hline \multirow[t]{3}{*}{ August } & 8 & $9 \cdot 9658$ & $0 \cdot 6136_{n}$ & $9 \cdot 9811$ & $0 \cdot 1014 n$ \\
\hline & 18 & $9 \cdot 9784$ & $0 \cdot 6257 n$ & $9 \cdot 9928$ & $0.1371_{n}$ \\
\hline & 28 & $9 \cdot 9893$ & $0 \cdot 6$ & $0 \cdot 0029$ & $454 n$ \\
\hline \multicolumn{2}{|c|}{ September 7} & $9 \cdot 9985$ & $0 \cdot 6416 n$ & 0.0119 & $0 \cdot 1794 \cdot n$ \\
\hline & 17 & 0.0077 & $0 \cdot 6412 n$ & 0.0200 & $0.1757 n$ \\
\hline & 27 & 0.0187 & $0 \cdot 6348 n$ & $0 \cdot 0303$ & $0.1542_{k b}$ \\
\hline \multirow[t]{2}{*}{ October } & 7 & $0.02+3$ & $4 n$ & 0.0353 & $0 \cdot 1096_{u}$ \\
\hline & 17 & 0.0329 & $0 \cdot 6$ & $0 \cdot 0+34$ & $0 \cdot 0362_{n}$ \\
\hline & 27 & & $5 n$ & 92 & $9 \cdot 9235_{n}$ \\
\hline & 0.0525 & $0.5414 n$ & 0.0617 & $9 \cdot 7485 n$ \\
\hline \multicolumn{2}{|c|}{$\begin{array}{r}\text { November } 6 \\
16\end{array}$} & 0.0637 & $0.505 .3 n$ & 0.0723 & $9 \cdot 4422_{n}$ \\
\hline & 26 & 0.0757 & $0 \cdot 4687 n$ & 0.0837 & $8 \cdot 0864 n$ \\
\hline \multicolumn{2}{|c|}{ December 6} & 883 & $0 \cdot 4354 n$ & 0.0957 & $9 \cdot 3233$ \\
\hline \multicolumn{2}{|c|}{16} & 0.0999 & $0 \cdot 4149 n$ & $0 \cdot 1067$ & $9 \cdot 5345$ \\
\hline \multicolumn{2}{|r|}{26} & 0.1133 & $0 \cdot 3940_{n}$ & $0 \cdot 1196$ & $9 \cdot 6726$ \\
\hline \multicolumn{2}{|r|}{36} & 0.1263 & $0 \cdot 3920_{n}$ & $0 \cdot 1321$ & $9 \cdot 6882$ \\
\hline \multicolumn{2}{|c|}{$k=$} & \multicolumn{2}{|c|}{-1.451} & \multicolumn{2}{|c|}{$\begin{array}{l}-1.693 \\
-0.693\end{array}$} \\
\hline
\end{tabular}

